\title{
BMJ Open Quality Reducing the rate of post-surgical urinary tract infections in orthopedic patients
}

\author{
Amit Thakker, ${ }^{1}$ Natasha Briggs, ${ }^{2}$ Azusa Maeda, ${ }^{1}$ Julie Byrne, ${ }^{2}$ \\ John Roderick Davey, ${ }^{2,3}$ Timothy D Jackson ${ }^{1,3}$
}

To cite: Thakker A, Briggs N, Maeda A, et al. Reducing the rate of post-surgical urinary tract infections in orthopedic patients. BMJ Open Quality 2018;7:e000177. doi:10.1136/ bmjoq-2017-000177

Received 3 August 2017 Revised 2 March 2018 Accepted 8 April 2018
Check for updates

${ }^{1}$ Division of General Surgery, University Health Network, Toronto, Ontario, Canada ${ }^{2}$ Division of Orthopedic Surgery, University Health Network, Toronto, Ontario, Canada ${ }^{3}$ Department of Surgery, Faculty of Medicine, University of Toronto, Toronto, Ontario, Canada

Correspondence to Dr Timothy D Jackson; timothy.jackson@uhn.ca

\section{ABSTRACT}

Urinary tract infection (UTI) is the fourth leading cause of healthcare-associated infections, with approximately $70 \%-80 \%$ being attributed to the inappropriate use of indwelling catheters. In many cases, indwelling catheters are used inappropriately without any valid indication, creating potentially avoidable and significant patient distress, discomfort, pain and activity restrictions, together with substantial care burden, cost and hospitalisation. In the Division of Orthopedic Surgery at Toronto Western Hospital (TWH), we identified UTI rate reduction as a quality improvement priority. Patients who underwent total hip and knee joint replacements and hip fracture repairs at TWH were monitored for the incidence of UTI and the usage of catheters. The data collected as part of the American College of Surgeons National Surgical Quality Improvement Program (ACS NSQIP) revealed UTI rate of $2.1 \%$ among 666 patients who were treated between January and June 2016. Data collected through a custom field in the ACS NSQIP workstation further revealed that indwelling catheters were overused, with $55.2 \%$ of patients receiving indwelling catheters in the same time period. These data were presented to the orthopaedic leadership group and surgeons at TWH in July 2016 to set the quality improvement target and create the working group. Nursing staff was provided education to strictly follow the institutional catheter-associated UTI prevention guidelines and change ideas based on the guidelines were implemented in July 2016. As a result, the rate of UTI decreased to $1.1 \%$ and the use of indwelling catheter decreased to $19.8 \%$ among 883 patients who were treated between July 2016 and March 2017. The study indicated that a systematic approach, engaging all front-line staff including nurse educators and nurse practitioners, helps to facilitate implementation of practice changes. We expect that ongoing reminders and education ensure that the changes are sustainable.

\section{PROBLEM}

Toronto Western Hospital (TWH) is a 272-bed teaching hospital within the University Health Network (UHN) in Toronto, Canada. Through participation in the American College of Surgeons National Surgical Quality Improvement Programme (ACS NSQIP), TWH was identified as having a higher incidence rate of postsurgical urinary tract infection (UTI) than other ACS NSQIP sites, particularly in the orthopaedic patients undergoing total hip and knee replacement surgeries and hip fracture repairs. UTI, commonly associated with an inappropriate use of indwelling urinary catheters, is a hospital-acquired infection that has a significant impact on patient outcomes, including the length of hospital stay, patient distress and mortality, as well as on healthcare expenditure. ${ }^{1}$ Further analysis of the TWH internal NSQIP database revealed a high frequency of indwelling urinary catheter use. Given that the overuse of indwelling urinary catheters contribute to the incidence of catheter-associated UTI (CAUTI), ${ }^{1}$ our aim was to reduce the rate of postsurgical UTI by $50 \%$ in the Division of Orthopedic Surgery at TWH in 9 months by implementing multifaceted strategies to reduce the unnecessary use of indwelling urinary catheters.

\section{BACKGROUND}

At UHN-TWH, a 'zero tolerance' for hospital-acquired infections is a driving force for this initiative. UTI is the fourth leading cause of healthcare-associated infections, ${ }^{2}$ with approximately $70 \%-80 \%$ being attributed to the inappropriate use of indwelling urinary catheters. ${ }^{1}$ CAUTI is associated with increased morbidity, mortality and prolonged length of stay. ${ }^{1}$ It is also associated with a significant cost to the healthcare system, with an estimated annual cost of approximately US\$38000 in the USA. ${ }^{3}$ Furthermore, the use of indwelling urinary catheters is associated with patient distress, discomfort, pain and limitations of daily activities. ${ }^{4}$

Studies suggest that the incidence of CAUTI can be reduced by avoiding unnecessary placement of indwelling urinary catheters and by promptly removing catheters when not required. ${ }^{5}$ Despite the knowledge about the strong linkage between the catheter use and the incidence of CAUTI, a survey of US hospitals demonstrated that more than 
half of the hospitals did not monitor patients for the presence of catheters, and close to $75 \%$ of the hospitals did not monitor the duration of catheter placements in patients. ${ }^{6}$ Given the financial and clinical consequences of CAUTI, strategies to promote appropriate catheter use are needed in order to reduce the rate of CAUTI.

A set of interventions known as the 'bladder bundle' was implemented by the collaborative initiative in the State of Michigan to reduce inappropriate use of indwelling urinary catheters over a 3-year period. ${ }^{7}$ The intervention provided education about the appropriate indications of catheter use and continued feedback on the performance of participating teams, and resulted in a significant reduction of inappropriate use of indwelling urinary catheters. ${ }^{8}$ Despite the successful implementation of the bundle strategy, these studies also highlighted difficulties to collect complete data over a long period of time in a large population of patients and to examine the influence of the intervention on the rate of UTI. A more recent study demonstrated that the implementation of strategies that promote appropriate catheter use resulted in the reduction of CAUTI rate at a single institution; however, the change in the practice of catheter use was not reported. ${ }^{9}$ In this current study, we performed a retrospective analysis of data on the use of indwelling urinary catheters and rate of UTI at our institution. The data were collected over a 14-month period in the ACS NSQIP database, assuring complete data collection and analysis for a large number of patients to examine the effectiveness of quality improvement interventions on reducing the rate of UTI.

\section{MEASUREMENT}

A retrospective analysis was performed using data from our internal ACS NSQIP database. The ACS NSQIP is a nationally validated database that captures 30 -day postsurgical clinical outcomes of patients, including the incidence of UTI. ${ }^{10}$ A custom field with a comment section about the use of indwelling urinary catheters was added to the ACS NSQIP workstation. Specifically, we collected information on the duration of catheter use and whether an intermittent or indwelling catheter was used. Our process measure was the frequency of indwelling urinary catheter use, and the outcome measure was the rate of UTI in patients who underwent elective hip and knee replacement surgery or hip fracture repair. Baseline UTI rate from January 2016 to June 2016 in the orthopaedic population at TWH $(\mathrm{n}=666)$ was $2.1 \%$, and indwelling urinary catheters were used in $55.2 \%$ of the patients. We planned to compare the baseline data with the data collected after implementation of change ideas, which was from July 2016 to March 2017.

\section{DESIGN}

The baseline data on the use of indwelling urinary catheters and NSQIP UTI rate was presented to the senior leadership group and orthopaedic surgeons to ensure their engagement to the quality improvement initiative. Data was also presented in a monthly orthopaedic quality meeting involving orthopaedic surgeons, nurse practitioners and nurse educators. Having members from the orthopaedic quality team and the inpatient unit was vital to ensure sustainability. A working group was formed to communicate the inappropriate use of indwelling urinary catheters. Guidelines relating to elimination of the unnecessary use of indwelling urinary catheters and prompt removal strategies were provided via education to all nursing staff. Based on the institutional CAUTI prevention guidelines, the change ideas were implemented in July 2016. Interventions included primarily education and a culture change of the nursing staff. The data on the use of indwelling urinary catheters and the rate of UTI was collected and presented in monthly orthopaedic quality meetings and in daily safety huddles after the changes were implemented.

\section{STRATEGY}

Following the analysis of the baseline data, the quality improvement initiative was implemented using the ' 4

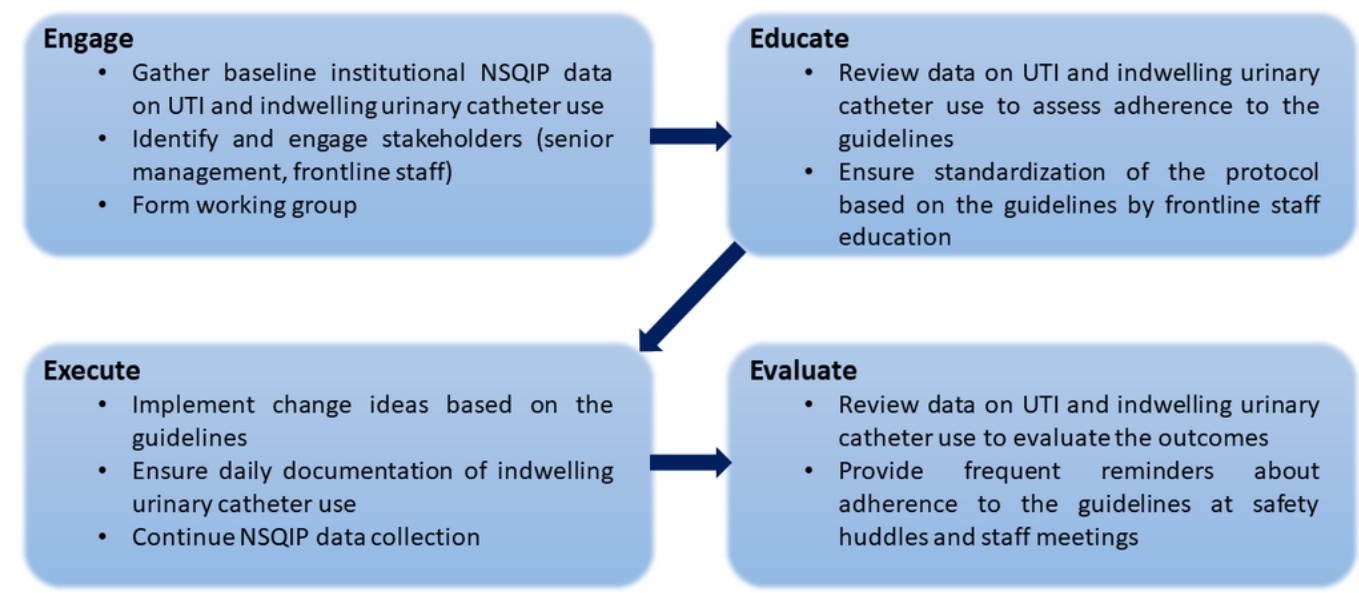

Figure 1 4E's approach implemented within the Division of Orthopedic Surgery at Toronto Western Hospital to reduce postsurgical urinary tract infections (UTI). NSQIP, National Surgical Quality Improvement Programme. 


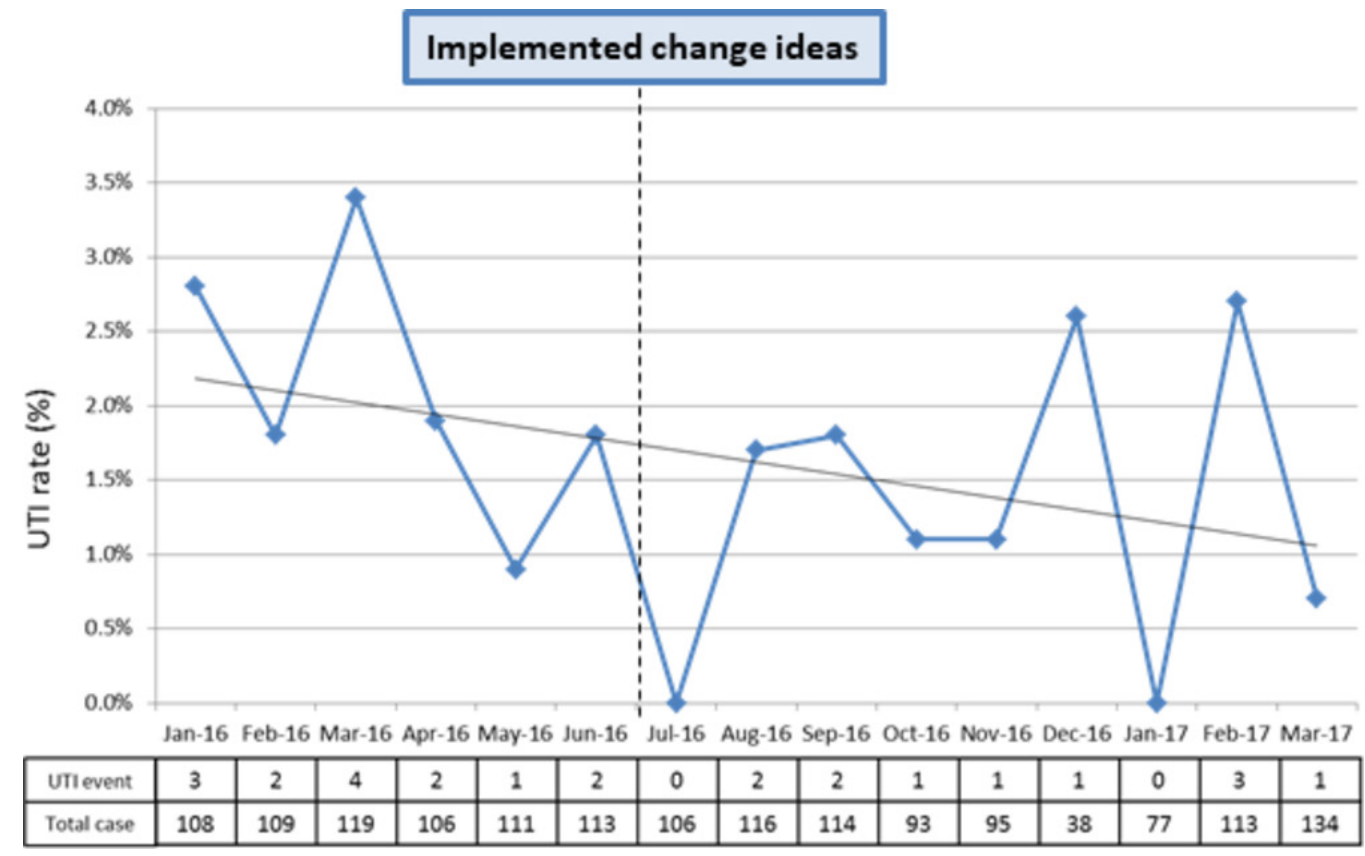

Figure 2 The rate of urinary tract infection between January 2016 and March 2017.

E's approach': engage, educate, execute and evaluate ${ }^{11}$ (figure 1).

\section{Engage}

We engaged senior management and front-line staff in this quality improvement initiative to ensure effective communication and to establish an improvement plan. Initially, data on the baseline UTI rate as well as the frequency of indwelling urinary catheter use on the inpatient unit was presented to senior management and surgeons. Further discussion involved the burden and extent of the problem by presenting known facts about CAUTI and a real story of a patient who acquired UTI on the nursing unit after a surgery. After getting approval from the senior management team, a working group was formed including nurses, a nurse educator and nurse practitioners to effectively implement the changes.

\section{Educate}

Previous to the intervention, providers were inclined to leave a catheter in situ if a patient with hip fracture had a low haemoglobin level, in case a blood transfusion with a diuretic was required. This concept was to limit the mobility of the patient during a transfusion. Furthermore, if a patient experienced urinary retention postoperatively, the inclination was to insert an indwelling urinary catheter for the night if unable to void after the initial intermittent catheterisation. However, these practices were not recommended in the institutional CAUTI prevention guidelines. Given low adherence to the existing institutional guidelines, nursing staff was educated about the guidelines to ensure adherence and to standardise the criteria for catheter use. This was a critical phase of the initiative, since the nurses initially reported that they were following the institutional CAUTI prevention guidelines until the baseline data were presented demonstrating inappropriate overuse of indwelling urinary catheter. During the intervention period, nurse practitioners worked closely with the staff nurses to ensure the change ideas were implemented effectively.

\section{Execute}

The following change ideas were implemented based on the institutional CAUTI prevention guidelines:

1. Offer bedpan to patient before scanning the bladder.

2. Use of intermittent catheterisation (in and out) when the bladder scan shows the postvoid residual volume of urine $>400 \mathrm{~mL}$.

3. Removal of an indwelling urinary catheter within 24 hours after surgery.

4. Daily documentation of indication if an indwelling urinary catheter is used.

\section{Evaluate}

Following the implementation of the strategies, data on the rate of UTI and the frequency of catheter use was collected on a monthly basis. The data were presented to the orthopaedic quality committee monthly, and the data were compared with the initial baseline data using run charts. To ensure sustainability, front-line staff was continually reminded about adhering to the CAUTI prevention guidelines in daily safety huddles and weekly staff meetings. The institutional CAUTI prevention guidelines were posted on the unit to make readily visible to all staff.

\section{RESULTS}

The average rate of UTI rate decreased from $2.1 \%$ between January and June 2016 to $1.1 \%$ between July 2016 and March 2017 (figure 2). Furthermore, the average rate of 


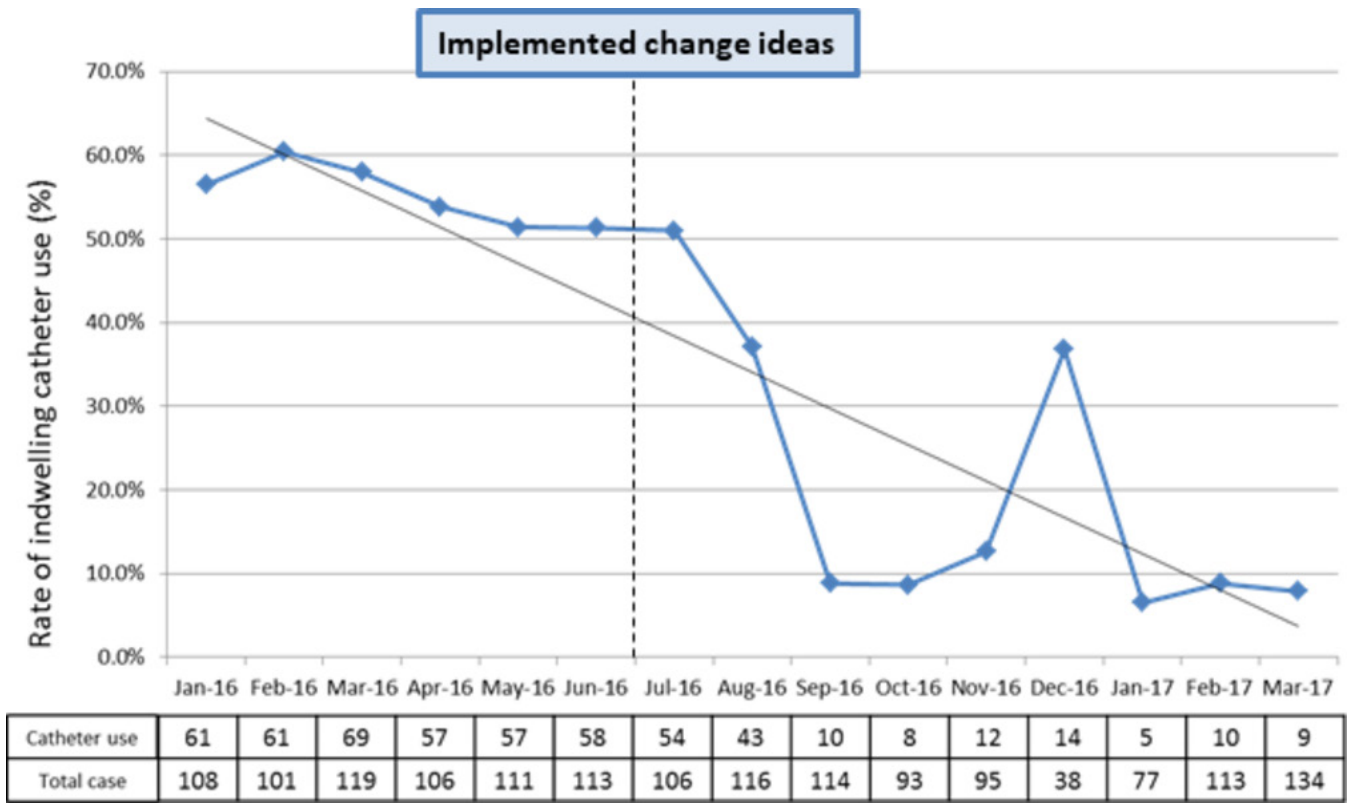

Figure 3 The frequency of indwelling catheter use between January 2016 and March 2017.

indwelling urinary catheter use decreased from $55.2 \%$ to $19.8 \%$ after implementation of the changes (figure 3). Through engagement of key team members, implementation of the institutional CAUTI prevention guidelines was successful and has demonstrated significant improvements. On the other hand, the average rate of intermittent catheterisation increased from $1 \%$ between January and June 2016 to 21.2\% between July 2016 and March 2017. Although the cost-effectiveness of the implemented strategies were not highlighted, it is obvious to ascertain that such a decrease in indwelling urinary catheters would be cost-effective. ${ }^{3}$

\section{LESSONS AND LIMITATIONS}

Our project highlighted the importance to engage staff at various levels to execute a quality improvement initiative. Initially, an email was sent to all nursing staff suggesting the need to reduce the number of indwelling urinary catheter insertions. However, this was not effective as the response rate was low. Group discussions had a greater impact in achieving compliance and staff participation through engagement. In addition to this, presentation of the initial baseline data to the senior management and orthopaedic surgeons' meeting was not well received as the data demonstrated unexpected amount of indwelling urinary catheter insertions that occurred in the inpatient unit. The NSQIP data were subsequently reviewed, and further data were captured to examine the duration of indwelling urinary catheters use. Having the extra data highlighted the importance of the problem and gave more perspective to the team, promoting engagement of the team. With support from the surgeons, senior management created a positive environment for change through collaboration with the nurse manager, nurse practitioner and nurse educator, enabling the front-line staff to own this quality improvement initiative. Presentation of the data on a monthly basis, frequent reminders through safety huddles and nurse educator's involvement assisted to reinforce the implemented strategies on the inpatient nursing unit. Our approach suggested that the front-line staff was receptive to change once they are fully engaged and see the value of the initiative to improve patient care.

Clinical nursing notes were used to record the relevant information. While useful, specific fields to provide the information were not provided, making it challenging for a data reviewer to locate the information needed. Furthermore, the indications for the use of indwelling urinary catheters were not captured consistently. We aim to improve the documentation system by developing an electronic platform, which would facilitate data collection and analysis.

The use of indwelling urinary catheter has decreased significantly as part of this initiative; however, the use of intermittent catheterisation has increased as it was being used more often to relieve postoperative urinary retention as per the CAUTI guideline. To reduce the need for catheterisation, we plan to perform a pilot study to eliminate the intraoperative use of intrathecal morphine, which is believed to cause postoperative urinary retention. ${ }^{12}$ With this pilot study, we expect to decrease the overall incidence of urinary retention, use of both indwelling and intermittent urinary catheters and ultimately the rate of UTI.

\section{CONCLUSION}

In the present study, we performed a retrospective analysis of data collected in the ACS NSQIP database for a total of 1549 patients who underwent joint replacements and repair of hip fractures at TWH between January 2016 and March 2017. The use of urinary catheters was documented as an additional variable in the ACS NSQIP database, enabling assessment of the influence 
of practice change to the clinical outcome. We demonstrated reduction of postsurgical UTI from $2.1 \%$ to $1.1 \%$, which is likely associated with the significant reduction of indwelling urinary catheter use. A systematic approach engaging all front-line staff helped to facilitate the implementation of this quality improvement initiative. Having a culture change on the inpatient unit was unexpected, yet favourable; the staff nurses acknowledged the impact of indwelling urinary catheter insertion and its risk for UTI and recognised the value for change. Regular presentation of the data further encouraged the team and ensured that the changes were implemented.

To ensure sustainability, the quality improvement team will continue to engage the nurse educator, nurse practitioner, providers and other members of the orthopaedic quality committee to incorporate the institutional CAUTI prevention guidelines in the nursing orientation curriculum. This, along with ongoing reminders and education through safety huddles and staff meetings, will ensure that the intervention is built into the current system and that the knowledge is transferred to new staff. Since the change ideas are generalisable, we anticipate that the intervention can be replicated in other units within the institution (eg, general surgery, neurosurgery) with additional culture change and engagement strategies from new stakeholders at each unit.

Acknowledgements The authors would like to thank Joe Brubaker and the nurses in the orthopaedics unit at TWH for their engagement in the project.

Contributors AT collected and analysed the data. AT, NB and JB led the project. AT, $\mathrm{NB}, \mathrm{AM}$ and TJ wrote the manuscript. All authors reviewed the manuscript. JRD and TJ supervised the project.

Funding The authors have not declared a specific grant for this research from any funding agency in the public, commercial or not-for-profit sectors.

Competing interests None declared.

Provenance and peer review Not commissioned; externally peer reviewed.
Open Access This is an Open Access article distributed in accordance with the Creative Commons Attribution Non Commercial (CC BY-NC 4.0) license, which permits others to distribute, remix, adapt, build upon this work non-commercially, and license their derivative works on different terms, provided the original work is properly cited and the use is non-commercial. See: http://creativecommons.org/ licenses/by-nc/4.0/

(C) Published by the BMJ Publishing Group Limited. For permission to use (where not already granted under a licence) please go to http://www.bmj.com/company/ products-services/rights-and-licensing/

\section{REFERENCES}

1. Nicolle LE. Catheter associated urinary tract infections. Antimicrob Resist Infect Control 2014;3:23.

2. Magill SS, Edwards JR, Bamberg W, et al. Multistate pointprevalence survey of health care-associated infections. N Engl J Med 2014;370:1198-208.

3. Kennedy EH, Greene MT, Saint S. Estimating hospital costs of catheter-associated urinary tract infection. J Hosp Med 2013;8:519-22.

4. Saint S, Lipsky BA, Baker PD, et al. Urinary catheters: what type do men and their nurses prefer? J Am Geriatr Soc 1999;47:1453-7.

5. Meddings J, Rogers MA, Krein SL, et al. Reducing unnecessary urinary catheter use and other strategies to prevent catheterassociated urinary tract infection: an integrative review. BMJ Qual Saf 2014;23:277-89.

6. Saint S, Kowalski CP, Kaufman SR, et al. Preventing hospitalacquired urinary tract infection in the United States: a national study. Clin Infect Dis 2008;46:243-50.

7. Saint S, Olmsted RN, Fakih MG, et al. Translating health careassociated urinary tract infection prevention research into practice via the bladder bundle. Jt Comm J Qual Patient Saf 2009;35:449-55.

8. Fakih MG, Watson SR, Greene MT, et al. Reducing inappropriate urinary catheter use: a statewide effort. Arch Intern Med 2012;172:255-60.

9. Taha H, Raji SJ, Khallaf A, et al. Improving catheter associated urinary tract infection rates in the medical units. BMJ Qual Improv Rep 2017;6:u209593.w7966.

10. Khuri SF, Henderson WG, Daley J, et al. Successful implementation of the department of veterans affairs' national surgical quality improvement program in the private sector: the patient safety in surgery study. Ann Surg 2008;248:329-36.

11. Pronovost PJ, Berenholtz SM, Needham DM. Translating evidence into practice: a model for large scale knowledge translation. BMJ 2008;337:a1714.

12. Tomaszewski D, Bałkota M, Truszczyński A, et al. Intrathecal morphine increases the incidence of urinary retention in orthopaedic patients under spinal anaesthesia. Anaesthesiol Intensive Ther 2014;46:29-33. 